Reprod. Nutr. Dévelop., 1986, 26 (6), 1295-1303.

\title{
Etude " in vitro " de quelques antibiotiques ionophores et de certains dérivés. I. Action sur les produits de la fermentation dans le rumen
}

\author{
S. CAFFAREL-MENDEZ $\left({ }^{*}\right)$, J.-P. JOUANY $\left({ }^{1}\right)$, Colette DEMUYNCK $\left({ }^{*}\right)$ \\ Laboratoire de la Digestion, I.N.R.A., \\ Theix 63122 Ceyrat, France. \\ (*) Laboratoire de Chimie Organique Biologique (UA 485) du CNRS, \\ UER de Recherche scientifique et technique, \\ Université de Clermont II, 63170 Aubière, France.
}

Summary. In vitro study of several ionophore antibiotics and some of their derivatives. I. Action on fermentation products in the rumen.

An in vitro study was conducted to test the action of different ionophore antibiotics and some of their derivatives on the end-products of rumen fermentation.

Nigericin and narasin, like monensin and lasalocid, increased the molar proportion of propionate in the V.F.A. mixture and decreased the proportion of acetate and especially that of butyrate. They had no action on total V.F.A. production. The effect observed with derivatives (lasalocid $\mathrm{O}$-acetyl or nigericin-O-acetyl) was generally less.

Gas production, chiefly methane, decreased with the addition of antibiotics. This result agrees with the stoichiometric reactions of carbohydrate fermentation in the rumen.

The amount of ammonia nitrogen fixed by bacteria was generally lowered by the addition of five antibiotics (nigericin, narasin, monensin, grisorixin and lasalocid) or two derivatives (lasalocid $\mathrm{O}$-acetyl and nigericin $\mathrm{O}$-acetyl), indicating a decrease in bacterial synthesis.

In contrast, calcimycin improved the butyrate production at the expense of propionate, and had no effect on gas composition or bacterial synthesis ; X $14547 \mathrm{~A}$ and alborixin had only a small effect on rumen fermentations.

These results, however, must be interpreted with care since the inocula used came from non-adapted sheep.

L'emploi d'additifs alimentaires pour manipuler les fermentations dans le rumen est désormais bien connu (Chalupa, 1980). Les antibiotiques, et parmi ceux-ci les antibiotiques ionophores, ont été les plus étudiés au cours de la dernière décennie (Durand, 1982 ; Bergen et Bates, 1984). Deux d'entre eux, la monensine et le lasalocide, sont aujourd'hui produits industriellement et utilisés comme anticoccidiens en aviculture. L'emploi de la monensine est également autorisé en Europe et aux Etats-Unis en tant que facteur de croissance chez les jeunes bovins. L'existence de résidus de monensine au niveau du foie de bovins

(') Demandes de tirés à part à J.-P. Jouany. 
traités (Donoho et al., 1978 ; Herberg et al., 1978 ; Donoho, 1984) ou de volailles (Donoho et Kline, 1967 ; Donoho, 1984) associée à une toxicité non négligeable de cette molécule (Wagner et al., 1983 ; Wardrope et al., 1983) rend difficile l'emploi de ce composé sans retrait préalable à l'abattage. Ainsi, et bien qu'ayant un seuil de toxicité moins élevé que celui du monensine (Weppelman et al., 1977), le lasalocide n'a pas été agréé comme additif dans les aliments du Ruminant. A l'avenir, l'innocuité des molécules proposées sera déterminante pour l'attribution de l'agrément par la Commission Européenne des Additifs. C'est pourquoi nous avons entrepris d'étudier in vitro l'action de divers ionophores sur les produits de fermentation dans le rumen et sur la quantité d'azote ammoniacal fixé par les bactéries, laquelle permet $d^{\prime}$ estimer la synthèse microbienne. Les résultats concernant chacune de ces molécules ont été comparés à ceux obtenus avec le monensine et le lasalocide, considérés comme molécules de référence. Les tests ont également porté sur certains dérivés de ces molécules qui ont été préparés par hémisynthèse dans le cadre d'une démarche plus générale pour tenter de réduire la toxicité des molécules originales. Ce travail est complété par une étude de leur action sur le transport in vitro des cations $\mathrm{Na}^{+}$et $\mathrm{K}^{+}$qui sera présentée dans une prochaine publication.

\section{Matériel et méthodes.}

Antibiotiques testés et doses. - L'étude a porté sur 8 antibiotiques ionophores : monensine A, lasalocide A, X 14547 A, nigéricine, grisorixine, alborixine, calcimycine, narasine et sur 3 de leurs dérivés : lasalocide $O$-acétyl, nigéricine $O$ acétyl, grisorixine O-méthyl. Chacun d'eux a été testé aux doses de 33 et $66 \mathrm{mg} / \mathrm{kg}$ d'amidon de blé ajoutés au milieu. Les dérivés ont été préparés dans le Laboratoire de Chimie Organique Biologique de I'Université de Clermont II.

Méthode " in vitro ». - Nous avons adapté la technique in vitro décrite par Jouany et Thivend (1986) afin d'étudier l'action des antibiotiques ionophores sur les principaux produits de la fermentation dans le rumen. L'inoculum a été préparé à partir de jus et de contenu de rumen prélevés sur 5 moutons adultes de race Texel munis chacun d'une canule de rumen et adaptés à recevoir la ration quotidienne suivante en 2 repas : $800 \mathrm{~g}$ de luzerne déshydratée et agglomérée, $200 \mathrm{~g}$ de paille de blé haché, $150 \mathrm{~g}$ d'orge broyée et agglomérée, $250 \mathrm{~g}$ de foin de prairie naturelle (ration sans adjonction d'antibiotique). Les animaux hébergeaient dans leur rumen une faune et une flore dites " conventionnelles ». Les prélèvements de contenu de rumen ont été effectués avant la distribution du repas du matin alors que les animaux étaient à jeun, y compris pour l'eau, depuis la veille à $17 \mathrm{~h}$.

Le fermenteur est constitué d'un flacon de 1 litre placé dans un bain-marie à agitation latérale et maintenu à $39^{\circ} \mathrm{C}$. Il est relié à une éprouvette remplie d'une solution saturée de $\mathrm{CaCl}_{2}$ renversée sur un bac contenant la même solution. Des tubes permettent de prélever du liquide et des gaz dans le milieu de fermentation. A la mise en route du fermenteur, nous avons introduit un inoculum composé de 
$200 \mathrm{ml}$ de salive artificielle ( $\left.{ }^{1}\right)$ saturée en $\mathrm{CO}_{2}$ à $40^{\circ} \mathrm{C}(\mathrm{pH}=6,8)$, homogénéisée avec $100 \mathrm{ml}$ de jus de rumen filtré et $100 \mathrm{ml}$ de contenu de rumen contenant les particules alimentaires.

L'apport d'énergie aux microorganismes a été assuré par l'addition, au milieu d'incubation, de $20 \mathrm{~g}$ d'amidon de blé et $5 \mathrm{~g}$ de glucose purs. Un apport d'azote $(186 \mathrm{mg})$ et de soufre $(212 \mathrm{mg})$ a également été fait dans chaque fermenteur sous forme de sulfate d'ammonium $(5 \mathrm{ml}$ d'une solution à $17,6 \% \mathrm{P} / \mathrm{V})$. Les fermenteurs dits "expérimentaux " ont reçu des antibiotiques à différentes doses ; les produits terminaux obtenus au cours des fermentations de 6 heures ont été comparés, pour chaque série d'incubation, à ceux d'un flacon témoin qui ne contenait pas d'antibiotique. Chaque série de fermentations est constituée de 3 fermenteurs :

$1=$ inoculum + amidon de blé $(20 \mathrm{~g})+$ glucose $(5 \mathrm{~g})+\left(\mathrm{NH}_{4}\right)_{2} \mathrm{SO}_{4}(186 \mathrm{mg}$ de N) :

2 = fermenteur $1+$ antibiotique $(33 \mathrm{ppm})$;

3 = fermenteur $1+$ antibiotique $(66 \mathrm{ppm})$.

Nous avons effectué 7 mesures pour chaque dose de monensine et de lasalocide, 3 répétitions pour la nigéricine, et 2 pour les autres antibiotiques et les dérivés d'antibiotiques.

Mesures. - Des échantillons liquides $(5 \mathrm{ml})$ ont été prélevés dans chaque fermenteur 0,5 et 6 heures après le début d'incubation et la valeur du $\mathrm{pH}$ du milieu a été mesurée immédiatement. Trois millilitres ont été utilisés pour déterminer la quantité d'acides gras volatils (AGV) et la composition de leur mélange selon la méthode de Jouany (1982). La concentration en azote ammoniacal $\left(\mathrm{N}-\mathrm{NH}_{3}\right)$ dans le liquide des fermenteurs a été déterminée à l'autoanalyseur Technicon par la méthode de Berthelot adaptée par Michel (1971). Des échantillons de gaz (4 ml) ont également été prélevés après 4 h 30 d'incubation. L'analyse de la composition du mélange gazeux a été faite par chromatographie en phase gazeuse selon la méthode décrite par Jouany et Senaud (1978).

La protéosynthèse nette qui a lieu entre les temps 0,5 et $6 \mathrm{~h}$ d'incubation a été assimilée à la quantité d'azote ammoniacal qui a disparu du fermenteur dans le même temps. La comparaison des résultats entre le fermenteur témoin et les fermenteurs expérimentaux permet de préciser l'effet de l'antibiotique, même si la valeur absolue du paramètre "synthèse bactérienne " obtenue par notre dispositif in vitro n'est pas rigoureusement exacte.

Le détail du calcul de la protéosynthèse bactérienne a été précisé par Jouany et Thivend (1986).

Les données ont été traitées statistiquement par un test de $F$, suivi du test non paramétrique de Mann et Whitney selon Snedecor et Cochran (1971).

Résultats (tabl. 1).

La valeur du $\mathrm{pH}$ du contenu inoculé a été de 6,80 à 7,00 à la mise en route des fermenteurs (TO). Les différences observées entre fermenteurs s'expliquent

(') Salive artificielle: $\mathrm{NaCl}=2,359 \mathrm{~g} ; \mathrm{KCl}=2,25 \mathrm{~g} ; \mathrm{MgCl}_{2} \cdot 6 \mathrm{H}_{2} \mathrm{O}=0,50 \mathrm{~g} ; \mathrm{CaCl}_{2} \cdot 2 \mathrm{H}_{2} \mathrm{O}=$ $0,36 \mathrm{~g}, \mathrm{NaHCO}_{3}=46,20 \mathrm{~g} ; \mathrm{NaHPO}_{4} \cdot 12 \mathrm{H}_{2} \mathrm{O}=35,62 \mathrm{~g} ; \mathrm{H}_{2} \mathrm{O}$ OSP 5 litres. 


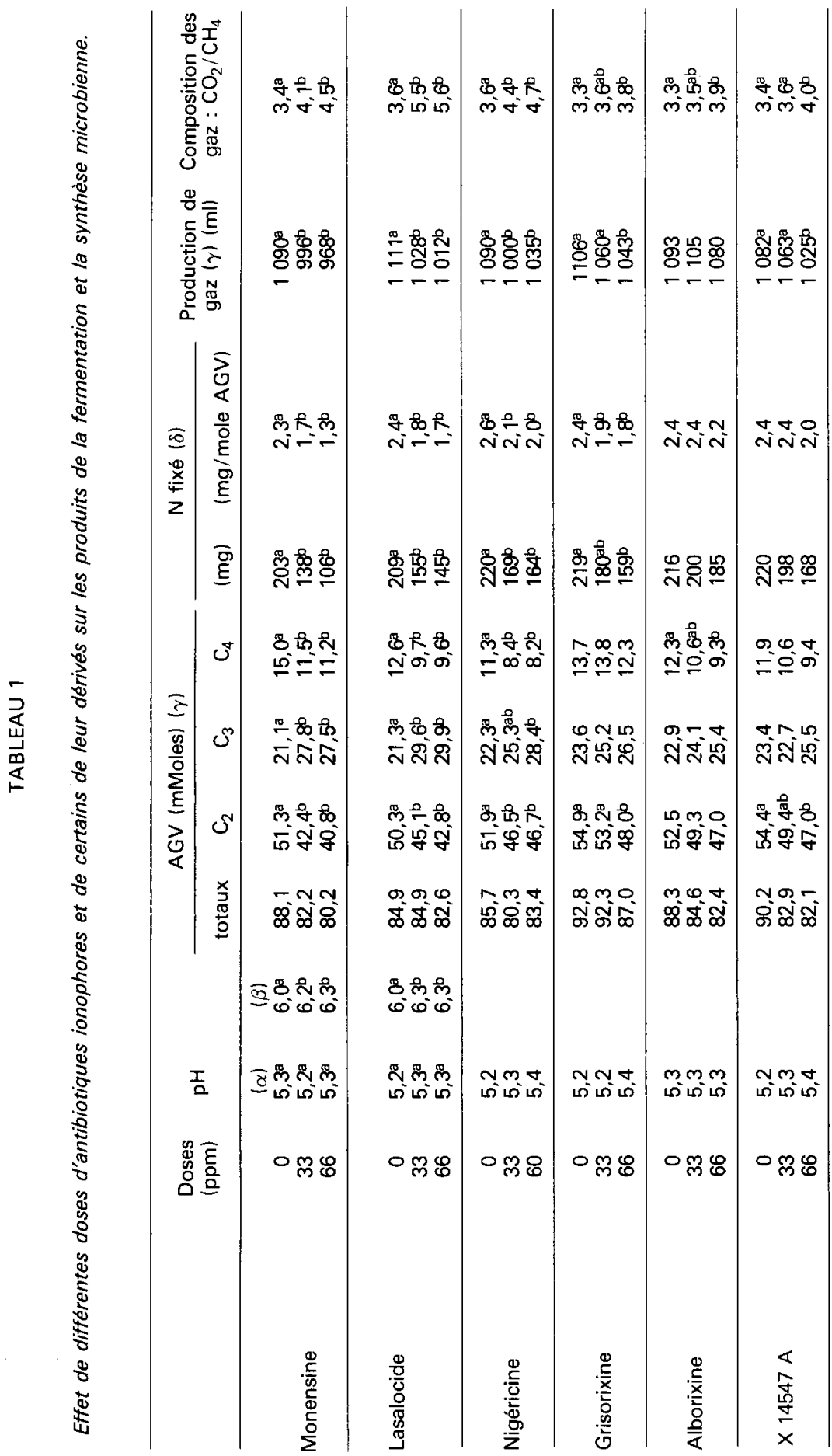




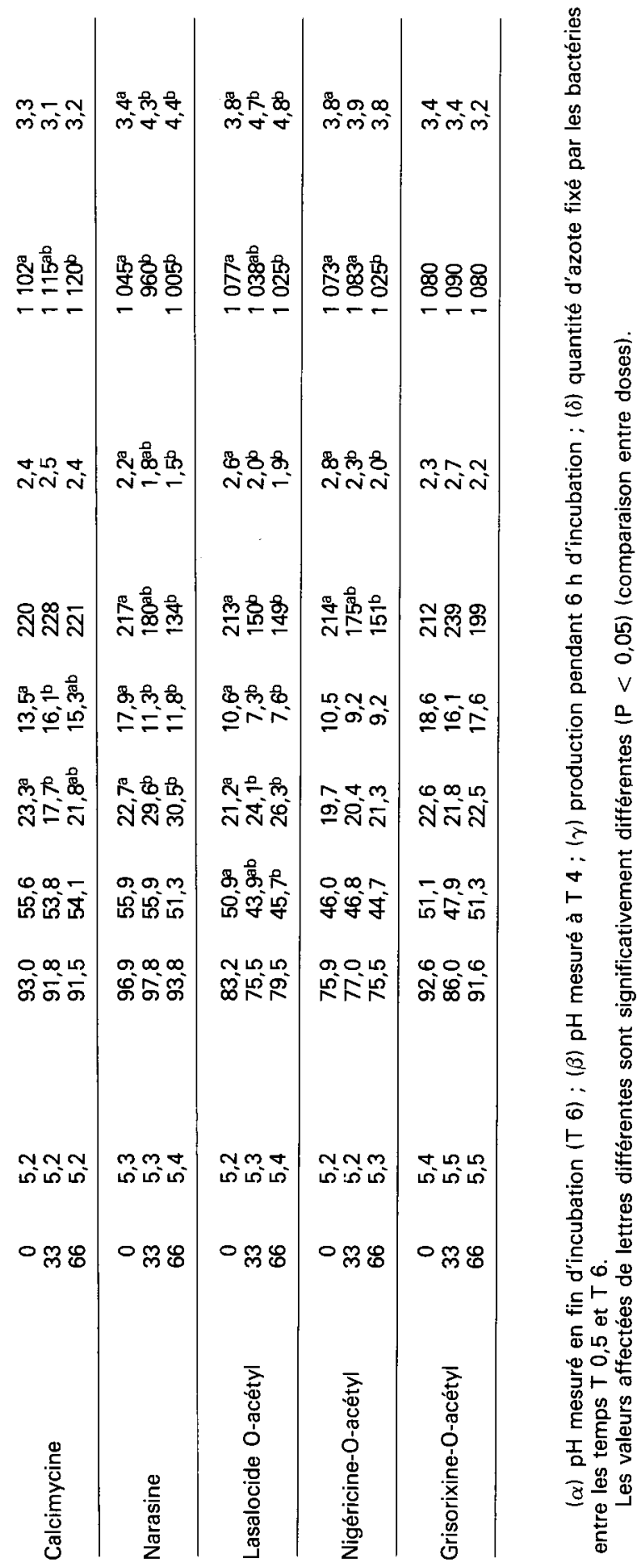


par les variations de la valeur du $\mathrm{pH}$ de la salive artificielle préparée extemporanément $(6,85<\mathrm{pH}<7,00)$, selon le temps de barbotage de $\mathrm{CO}_{2}$. Bien que les valeurs moyennes du $\mathrm{pH}$ en fin d'incubation aient été faibles $(5,2$ à 5,5), l'effectif moyen des protozoaires vivants était voisin de celui mesuré à la mise en route des fermenteurs ( $3 \times 10^{5}$ protozoaires par $\mathrm{ml}$ de contenu de fermenteur).

Mise à part une augmentation significative de la valeur du $\mathrm{pH}$ au temps $4 \mathrm{~h}$ observée dans les fermenteurs contenant la monensine ou le lasalocide, l'addition des différents antibiotiques testés n'a pas eu d'effet sur l'évolution cinétique du $\mathrm{pH}$ au cours des fermentations. Corrélativement, la quantité totale d'AGV produits pendant $6 \mathrm{~h}$ de fermentation n'a pas été influencée par la présence des antibiotiques. En revanche, la composition du mélange d'AGV a fréquemment évolué. Parmi les produits testés, nous distinguerons 3 groupes :

- dans l'un d'eux (comprenant la monensine, le lasalocide, le lasalocide O-acétyl, la nigéricine et la narasine) nous avons observé une augmentation significative de la production d'acide propionique au détriment de celle des acides acétique et butyrique. Le même effet a été observé avec la grisorixine et l'alborixine, mais à un degré moindre, puisque le seuil de signification $(P<0,05)$ n'a pas été atteint. - le deuxième groupe (qui comprenait le $X 14547 \mathrm{~A}$, la nigéricine O-acétyl et la grisorixine O-méthyl) n'a eu aucune action à l'égard de la production d'acide propionique ; les modifications induites sur les acides acétique ou butyrique ont été négligeables, ou bien significatives seulement à la dose de 66 ppm.

- enfin, la calcimicyne a eu un effet inverse de celui des autres antibiotiques puisqu'elle a provoqué une réduction significative de la production d'acide propionique au profit de celle de l'acide butyrique ; l'effet sur l'acide acétique a été nul. Dans la plupart des cas, le seuil de signification $(P<0,05)$ a été atteint avec la dose de $33 \mathrm{ppm}$.

La production gazeuse totale a été significativement diminuée par l'addition de monensine, lasalocide, nigéricine, narasine, grisorixine, $X 14547 \mathrm{~A}$ ainsi que par le lasalocide 0 -acétyl. Pour les 4 derniers, le seuil de signification $(P<0,05)$ n'a été atteint qu'à la dose de 66 ppm. Les autres antibiotiques ou dérivés n'ont pas eu d'effet sur la production gazeuse.

La composition du mélange gazeux provenant des fermenteurs expérimentaux, à l'exception des fermenteurs ayant reçu la calcimycine, la nigéricine 0 acétyl et la grisorixine $\mathrm{O}$-acétyl, a été différente de celle issue des fermenteurs témoins. En présence de monensine, lasalocide, nigéricine, narasine, lasalocide 0 acétyl, le rapport $\mathrm{CO}_{2} / \mathrm{CH}_{4}$ a très fortement augmenté $(\mathrm{P}<0,001)$; l'effet était significatif dès la dose de $33 \mathrm{ppm}$. La valeur du rapport $\mathrm{CO}_{2} / \mathrm{CH}_{4}$ a également été accrue par l'addition de grisorixine, d'alborixine ou de $\times 14547 \mathrm{~A}$, mais la signification de l'effet n'a été atteinte $(P<0,05)$ qu'à la dose de 66 ppm.

La synthèse d'azote microbien, estimée par la quantité d'azote ammoniacal fixée par les bactéries entre les temps 0,5 et 6 heures de fermentations, a généralement été réduite par l'addition d'antibiotiques dans les fermenteurs. Cette réduction n'a pas été significative en présence d'alborixine et de calcimycine. Le seuil de signification $(P<0,05)$ n'a été atteint qu'à la dose de 66 ppm, après l'addition de grisorixine, de $\times 14547$ A et de narasine. La transformation des molécules originales d'antibiotiques en dérivés a entraîné une diminution de leur 
effet sauf dans le cas du lasalocide O-acétyl. L'efficacité de la synthèse microbienne, exprimée en quantité d'azote microbien synthétisé par mole d'AGV produite, a été significativement diminuée par l'addition de la monensine, du lasalocide et de son dérivé, de la nigéricine et de son dérivé, et de la grisorixine à la dose de $33 \mathrm{ppm}$. La narasine et le X 14547 A n'ont eu d'effet qu'à la dose de $66 \mathrm{ppm}$. En revanche, la synthèse microbienne a été significativement augmentée par la présence de calcimycine. L'alborixine et la grisorixine O-méthyl n'ont pas modifié ce paramètre.

\section{Discussion et conclusion.}

Le dispositif de fermentation utilisé nous a permis de vérifier in vitro l'action maintenant bien connue de la monensine et du lasalocide sur les produits de la fermentation et sur l'activité microbienne dans le rumen. II nous a également permis de montrer que la nigéricine et la narasine ont toutes deux amélioré la production de propionate dans des proportions voisines de celles mesurées en présence de monensine ou de lasalocide. Leur effet dépressif sur la production de méthane s'apparente également à celui des deux molécules de référence précitées, ce qui est conforme aux réactions stœchiométriques associant la production des gaz à celle des AGV dans le rumen (Hungate, 1966). Nos tests ont donc montré que ces deux molécules pourraient avoir une application en tant que facteur de croissance. Une expérience réalisée in vivo avec la narasine (Gay et al., 1979) a d'ailleurs montré que chez des bœufs recevant une dose de $16 \mathrm{ppm}$ de cet antibiotique dans leur ration, les modifications dans le rapport acétate/propionate étaient proches de celles mesurées dans notre étude.

La transformation de la fonction- $\mathrm{OH}$ du cycle terminal de la nigéricine, du lasalocide et de la grisorixine en éther méthylé ou acétylé a généralement provoqué une diminution de l'activité des molécules témoins à l'égard des fermentations dans le rumen. Seul le lasalocide O-acétyl a conservé une action significative sur les principaux paramètres fermentaires. La diminution de la quantité d'azote ammoniacal fixé par les bactéries qui a été observée après l'introduction de ces molécules, à l'exception de l'alborixine, de la calcimycine et de la grisorixine Ométhyl, est probablement la conséquence d'une réduction de la synthèse microbienne dans les fermenteurs expérimentaux. Ce résultat peut expliquer l'action négative de ces additifs sur la croissance d'animaux recevant des régimes riches en azote non protéique comme l'ont montré Short (1978), Oltjen et al. (1977) et Coombe et al. (1979), avec la monensine. La diminution de l'efficacité de la synthèse microbienne (exprimée par mole d'AGV produite) que nous avons mise en évidence à la dose de $33 \mathrm{ppm}$ avec la monensine, le lasalocide, la nigéricine et la narasine, traduisent un découplage des fermentations comme cela a été discuté par Van Nevel (1977). La modification chimique du lasalocide et de la nigéricine a entraîné la suppression de cet effet.

La calcimycine a eu un comportement original puisqu'elle a entraîné une orientation des fermentations différente de celle observée avec les autres antibiotiques. L'augmentation de la production de butyrate au détriment du propionate permet d'envisager l'emploi de cette molécule comme additif alimentaire chez la 
vache laitière. Dans le même temps, elle n'a pas eu d'effet inhibiteur sur la fixation de l'azote ammoniacal par les bactéries du rumen ce qui, en théorie, rend son emploi possible même avec des rations riches en azote non protéique. Mais la toxicité élevée de cette molécule est un obstacle à son utilisation pratique comme additif alimentaire chez le Ruminant.

Nos résultats montrent que les molécules sont généralement actives à la dose de $33 \mathrm{ppm}$ et que le passage à $66 \mathrm{ppm}$ modifie peu les paramètres fermentaires et la quantité d'azote fixée par les bactéries. Toutefois, la grisorixine et le $X 14547 \mathrm{~A}$ n'ont eu un effet significatif sur les paramètres mesurés qu'à la seule dose de $66 \mathrm{ppm}$. Or, dans la pratique, la monensine est distribuée au Ruminant à raison de 20 à $40 \mathrm{mg} / \mathrm{kg}$ d'aliment, ce qui correspond en moyenne à la dose de 33 ppm utilisée dans notre étude. Les antibiotiques dont l'activité n'apparaît qu'à 66 ppm ont donc peu d'intérêt, à moins que la quantité de résidus présents, en particulier dans le foie, et que la toxicité de ces molécules, soient faibles.

Enfin, il faut préciser que les mesures ont été effectuées in vitro avec des inocula prélevés sur des animaux non adaptés à recevoir ces antibiotiques. L'application in vivo de ces résultats ne peut s'envisager qu'avec précaution puisque plusieurs travaux ont montré que l'orientation des fermentations diverge suivant qu'ils ont été obtenus à partir d'animaux adaptés ou non à recevoir ces molécules (Short, 1978 ; Herod et al., 1979 ; de Jong et Berschauer, 1983). Les petites quantités de ces divers antibiotiques qui ont été produites au laboratoire (quelques dizaines de milligrammes) n'ont pas permis d'envisager leur distribution aux animaux donneurs d'inocula pendant une période de plusieurs semaines à la dose de $30 \mathrm{ppm}$. C'est pourquoi l'expérimentation avec des animaux adaptés n'a pas été possible au cours de cette étude.

Reçu en mai 1986. Accepté en septembre 1986.

Remerciements. - Les auteurs remercient Mlle Paulette Journaix, Mme Marie-Paule Girard ainsi que MM. L. L'Hotelier, M. Fabre, P. Pichon pour leur assistance technique.

\section{Références}

BERGEN W. G., BATES D. B., 1984. lonophores : their effect on production efficiency and mode of action. J. anim. Sci, 58, 1465-1483.

CAFFAREL-MENDEZ S., 1984. Contribution à l'étude du mode d'action des antibiotiques ionophores carboxyliques. I. Etude du mécanisme de transport des ions alcalins. II. Influence sur les paramètres fermentaires du rumen mesuré in vitro. Th. Doct. Ing. Univ. Clermont II - $\mathrm{n}^{\circ} \mathrm{d}^{\prime}$ ordre 134, 206 pages.

CHALUPA W., 1980. Chemical control of rumen microbial metabolism, 325-347. In RUCKEBUSH Y., THIVEND P. Digestive physiology and metabolism in ruminants, M.T.P. Press, Lancaster.

COOMBE J. B., DINIUS D. A., GOERING H. K., OLTGEN R. R., 1979. Wheat straw-urea diets for beef steers : alkali-treatment and supplementation with protein, monensin and a feed intake stimulant. J. anim. Sci, 48, 1223-1233.

DONOHO A. L., KLINE R. M., 1967. Monensin, a new biologically active compound. VII. Thinlayer bioautographic assay for monensin in chick tissues, 763-766. In HOBBY G. L. Antimicrobial agents and chemotherapy, Amer. Soc. Microbiol.-U.S.A. 
DONOHO A. MANTHEY J., OCCOLOWITZ J., ZORNES L., 1978. Metabolism of monensin in the steer and rat. J. agric. Food Chem., 26, 1090-1095.

DONOHO A. L., 1984. Biochemical studies on the fate of monensin in animals and in the environment. J. anim. Sci., 58, 1528-1539.

DURANT M., 1982. Orientation du métabolisme de la panse au moyen des additifs. Ann. Zootech., 31, 47-76.

GAY N., OSHIDARI S., BRADLEY N. W., BROWN H., MUIR W., BOLING J. A., 1979. Influence of a monensin derivative (narasin) on finishing steers. J. anim. Sci, 49, 30 (Abst.).

HERBERG R., MANTHEY J., RICHARDSON L., COOLEY C., DONOHO A. L., 1978. Excretion and tissue distribution of ${ }^{14} \mathrm{C}$ monensin in cattle. J. agric. Food. Chem., 26, 1087-1090.

HEROD E. L., BARTLEY E. E., DAVIDOVITCH A., BECHTLE R. M., SAPIENZA D. A., BRENT B. E., 1979. Effect of adaptation to monensin or lasalocid on rumen fermentation in vitro and the effect of these drugs on heifer growth and feed efficiency. J. anim. Sci., 49 Suppl. 1, 374 (Abstr.).

HUNGATE R. E., 1966. The rumen and its microbes. Acad. Press, New York, San Francisco, London.

JONG A. de, BERSCHAUER F., 1983. Adaptation effects of ionophores on rumen fermentation. $S$. Afric. J. anim. Sci, 13, 67-70.

JOUANY J. P., SENAUD J., 1978. Utilisation du monensin dans la ration des ruminants. II. Effets sur les fermentations et la population microbienne du rumen. Ann. Zootech., 27, 61-74.

JOUANY J. P., 1982. V.F.A. and alcohol determination in digestive contents, silage juice, bacterial cultures and anaerobic fermentor contents. Sci. Aliments, 2, 131-144.

JOUANY J. P., THIVEND P., 1986. In vitro effects of avoparcin on protein degradability and rumen fermentation. Anim. Feed Sci. Technol., 15, 215-229.

MICHEL M. M., 1971. Analyse quantitative de quelques substances azotées et glucidiques en milieu biologique; essai de rationalisation. Th. Univ. Clermont-Ferrand- $\mathrm{n}^{\circ} \mathrm{d}^{\prime}$ ordre $43,79 \mathrm{pp}$

OLTJEN R. R., DINIUS D. A., GOERING H. K., 1977. Performance of steers fed crop residues supplemented with non protein nitrogen, minerals, protein and monensin. J. anim. Sci., 45, $1442-1452$.

SHORT D. E., 1978. Rumen fermentation and nitrogen metabolism as affected by monensin in vitro. Ph. D. Thesis, University of Illinois, Urbana. Champaign, USA.

SNEDECOR G. W., COCHRAN W. G., 1971. Méthodes statistiques. 6e édition. A.C.T.A. ed. Paris. VAN NEVEL C. J., DEMEYER D. I., 1977. Effect of monensin on rumen metabolism in vitro. Appl. environ. Microbiol., 34, 251-257.

WAGNER D. D., FURROW R. D., BRADLEY B. D., 1983. Subchronic toxicity of monensin in broiler chickens. Vet. Path., 20, 353-359.

WARDROPE D. D., Mac LEOD N. S., SLOAN J. R., 1983. Outbreak of monensin poisoning in cattle. Vet. Rec., 112, 560-561.

WEPPELMAN R. M., OLSON G., SMITH D. A., TAMAS T., VAN IDERSTINE A., 1977. Comparison of anticoccidial efficacy, resistance and tolerance of narasin, monensin and lasalocid in chicken battery trials. Poultr. Sci., 56, 1550-1559. 